\title{
Accuracy in Resolving the First Hydration Layer on a Transition Metal Oxide Surface: Experiment (AP-XPS) \& Theory
}

\author{
Daniel J. Aschaffenburg, $\chi$ Seiji Kawasaki, $\chi$ Chaitanya Das Pemmaraju, ${ }^{\ddagger}$ and Tanja Cuk $\chi, \mid, *$ \\ ${ }^{\chi}$ Chemical Sciences Division, Lawrence Berkeley National Laboratory, Berkeley, CA, United States \\ $\$$ Theory Institute for Materials and Energy Spectroscopies, SLAC National Accelerator Laboratory, Menlo Park, \\ California 94025, United States \\ Department of Chemistry and Renewable and Sustainable Energy Institute, University of Colorado, Boulder, \\ Boulder, Colorado 80303, United States
}

\section{Experimental Details: UHV Conditions after Surface Cleaning}
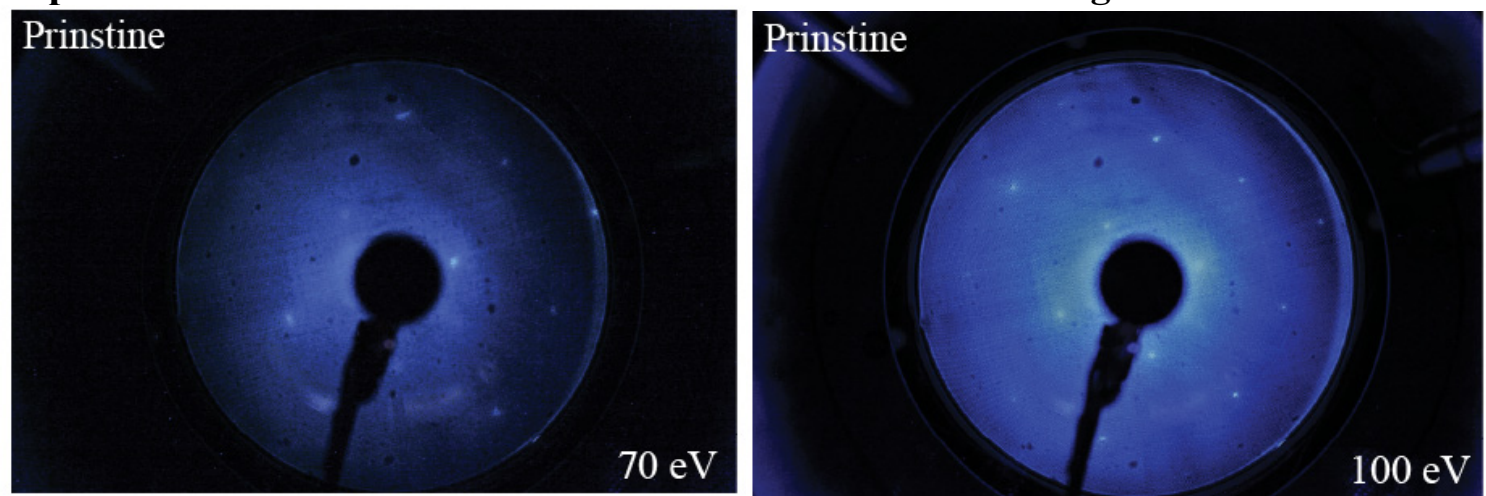

Figure S1. LEED images of a $\mathrm{SrTiO}_{3}(100)$ after UHV cleaning exhibiting the pattern for a (100) surface. The images show a typical $\mathrm{P}(1 \mathrm{x} 1)$ structure of a (100) orientation of a cubic perovskite $(1)$. When the sample has also been cleaned by $600^{\circ} \mathrm{C}$ under UHV conditions, only the spots associated with the structure rather than $\mathrm{O}$ vacancy defects appear; this is systematically reported in (2) that also contains similar images as that shown above. While some background is present in the LEED images, which could either be due to the sample or the well-utilized instrument, this does not preclude the assessment of a predominant $\mathrm{P}(1 \times 1)$ structure.

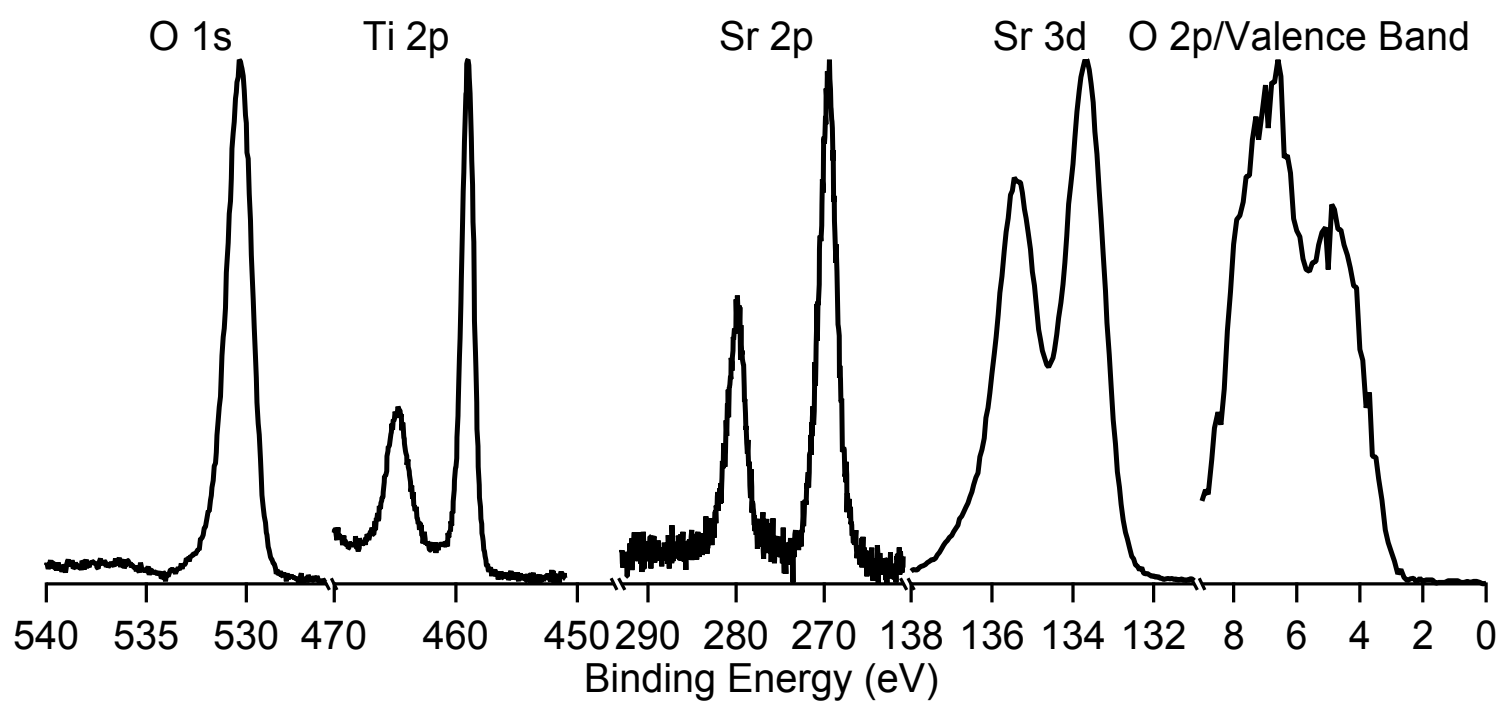


Figure S2. UHV XPS spectra of $\mathrm{SrTiO}_{3}(100)$ with $\mathrm{TiO}_{2}$ terminated surface. After UHV annealing, the sample was transferred from the preparation chamber to the spectroscopy chamber. XPS data were collected for $\mathrm{O} 1 \mathrm{~s}$, Ti 2p, C 1s/Sr 2p, Sr 3d, and O 2p (valence band, VB) core levels at an incident X-ray energy of $735 \mathrm{eV}$. STO showed no detectable amount of surface carbon contamination after UHV annealing (shown below in Fig. S9 and S10).

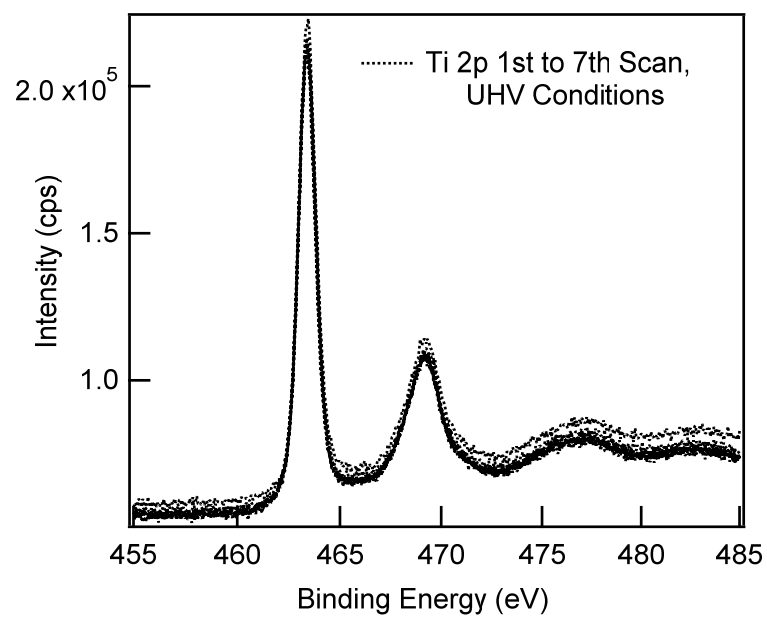

Figure S3. Before exposure to ambient pressure, damage tests were performed to assess potential $\mathrm{X}$-ray damage. No changes in the $\mathrm{Ti} 2 \mathrm{p}$ spectra (indicative of $\mathrm{Ti}^{3+}$ which arise from defect states/Ovacancies) were observed. There is also no clear shoulder in the $\mathrm{Ti} 2 \mathrm{p}$ data indicative of vacancies.
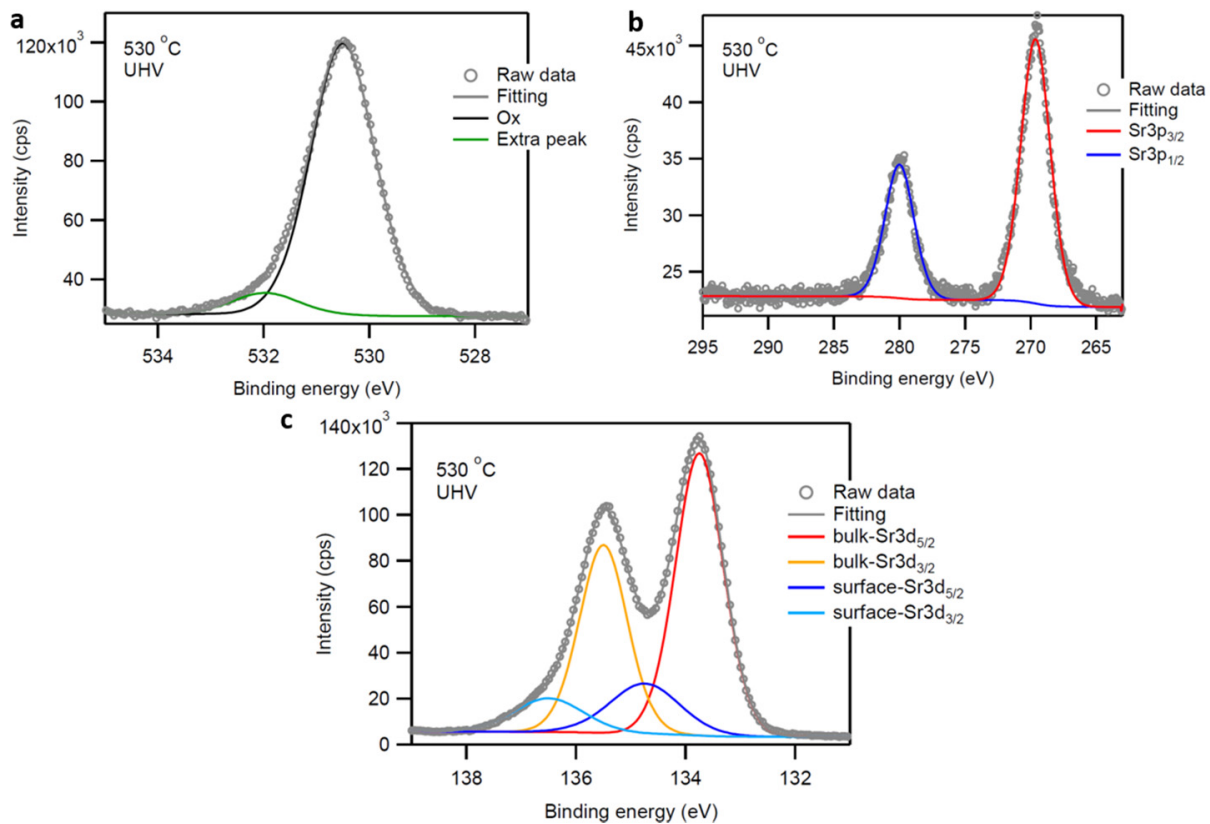

Figure S4. XPS data and fits at UHV and high temperature $\left(530^{\circ} \mathrm{C}\right)$ conditions (a) $\mathrm{O} 1 \mathrm{~s}$ peak with a fit that includes the intrinsic defect peak to accommodate the asymmetry on the higher binding energy side (b) XPS data in C1s and Sr2p range, showing the lack of carbon in the 282-295 eV range and the clear Sr2p peaks. (c) Sr3d peaks are best fit with two peaks each, suggesting a separate surface component. This surface component within Sr 3d spectra has been previously attributed to F contamination of the STO (100 surface) $(3,4)$ after the surface preparation with an 
HF etch and appears increasingly with higher emission angles from the normal (here, $42^{\circ}$ ). The data shown should be compared with that taken at $0^{\circ}$ and $90^{\circ}$ in Fig. $2 \mathrm{~b}$ of Chambers et. al.(3).

Fitting of XPS O 1s core level spectra

\begin{tabular}{|c|c|c|c|c|c|c|c|c|}
\hline $\begin{array}{l}\mathrm{P}(\mathrm{H} 2 \mathrm{O}) \\
(\mathrm{m})\end{array}$ & $\mathrm{T}\left(\mathrm{C}^{\circ}\right)$ & RH (\%) & x Area & $\mathrm{OH}$ Area & CO3 Area & H2O Area & Defect Area & Defect/Ox \\
\hline 30 & 596 & 0 & 381408 & 0 & 0 & 0 & 30512.6 & 0.08 \\
\hline 30 & 349.7 & $2.38 \mathrm{E}-05$ & 409797 & 14480.3 & 0 & 0 & 32783.8 & 0.08 \\
\hline 30 & 300.4 & $4.55 \mathrm{E}-05$ & 416367 & 19273 & 0 & 0 & 33309.3 & 0.08 \\
\hline 30 & 250.1 & $9.86 \mathrm{E}-05$ & 407715 & 20362.7 & 0 & 0 & 32617.2 & 0.08 \\
\hline 30 & 200.4 & 0.000251 & 357004 & 28171.2 & 12378.3 & 2384.6 & 28560.3 & 0.08 \\
\hline 30 & 174.4 & 0.000447 & 247344 & 18663.8 & 8789.9 & 1514 & 19787.5 & 0.08 \\
\hline 30 & 145 & 0.000946 & 339416 & 21894.8 & 12578.9 & 2144.1 & 27153.2 & 0.08 \\
\hline 30 & 125.2 & 0.001684 & 320411 & 25737.8 & 16000.3 & 6469 & 25632.9 & 0.08 \\
\hline 30 & 100.3 & 0.003842 & 287375 & 26983.1 & 10000 & 16792.9 & 22990 & 0.08 \\
\hline 30 & 80.3 & 0.008211 & 263318 & 44156.1 & 15000 & 13201.4 & 21065.5 & 0.08 \\
\hline 30 & 60.3 & 0.019502 & 243310 & 66942.2 & 20367.1 & 32909.3 & 19464.8 & 0.08 \\
\hline 30 & 40.6 & 0.051787 & 172332 & 112339 & 20000 & 59670.5 & 13786.5 & 0.08 \\
\hline 30 & 20.8 & 0.160867 & 196495 & 103030 & 30000 & 85983.7 & 15719.6 & 0.08 \\
\hline 30 & 10.8 & 0.305402 & 182472 & 100139 & 30000 & 74987.1 & 14597.7 & 0.08 \\
\hline 30 & 5.8 & 0.429098 & 190612 & 97129.3 & 30000 & 80281.1 & 15248.9 & 0.08 \\
\hline 100 & 565 & 0 & 189528 & 0 & 0 & 0 & 13266.9 & 0.07 \\
\hline 101 & 349.8 & 8.01E-05 & 187290 & 8380.2 & 0 & 0 & 14983.2 & 0.08 \\
\hline 101 & 299.7 & 0.000155 & 184640 & 8711.8 & 0 & 0 & 14771.2 & 0.08 \\
\hline 101 & 249.2 & 0.000337 & 170916 & 17117.3 & 4196.1 & 1034.8 & 13673.3 & 0.08 \\
\hline 101 & 200.4 & 0.000844 & 167834 & 13401.7 & 6051.6 & 3080.7 & 13426.8 & 0.08 \\
\hline 101 & 175.6 & 0.001462 & 150492 & 16373.8 & 6020.3 & 2978.4 & 13379.7 & 0.088906 \\
\hline 100 & 142.7 & 0.003362 & 119656 & 16098.2 & 7000 & 3200.2 & 9572.5 & 0.08 \\
\hline 100 & 124.4 & 0.005754 & 118136 & 15914.9 & 6750 & 5431.6 & 9450.9 & 0.08 \\
\hline 100 & 100.6 & 0.01267 & 112652 & 21404 & 10834.8 & 6292.3 & 9012.2 & 0.08 \\
\hline 99 & 78.8 & 0.028801 & 55611.4 & 18676.1 & 8000 & 6234.6 & 4448.9 & 0.08 \\
\hline 100 & 60.5 & 0.064408 & 35576.5 & 19493.9 & 9042.9 & 11214.5 & 2846.1 & 0.079999 \\
\hline 99 & 40.5 & 0.171806 & 78739.2 & 43338.9 & 23000 & 27421.6 & 6299.1 & 0.08 \\
\hline 99 & 20.7 & 0.534143 & 75202.8 & 39651.4 & 17273.2 & 30139.2 & 6016.2 & 0.08 \\
\hline 99 & 10.8 & 1.00783 & 68648.6 & 39615.5 & 26000 & 26970.3 & 5491.9 & 0.08 \\
\hline 99 & 5.5 & 1.44585 & 54059.8 & 28181.1 & 12000 & 28363.7 & 4324.8 & 0.08 \\
\hline 500 & 525 & 0 & 330039 & 0 & 0 & 0 & 26403.1 & 0.08 \\
\hline 501 & 500 & 0 & 279397 & 0 & 0 & 0 & 22351.8 & 0.08 \\
\hline 500 & 400.4 & 0 & 184476 & 0 & 0 & 0 & 14758.1 & 0.08 \\
\hline 499 & 349.5 & 0.000397 & 282849 & 16482.7 & 0 & 0 & 22627.9 & 0.08 \\
\hline 498 & 300.6 & 0.000752 & 278600 & 14982.6 & 0 & 0 & 22288 & 0.08 \\
\hline 501 & 250.6 & 0.001633 & 265659 & 22971.7 & 10000 & 0 & 21252.7 & 0.08 \\
\hline 501 & 224 & 0.002622 & 258781 & 28300.8 & 13489.4 & 3293.6 & 20702.5 & 0.08 \\
\hline 500 & 200.6 & 0.004159 & 242462 & 24834.8 & 13000 & 6333.7 & 19397 & 0.08 \\
\hline 498 & 175.4 & 0.007242 & 248024 & 32119.4 & 13000 & 23875.9 & 19842 & 0.08 \\
\hline 500 & 150.6 & 0.013547 & 228245 & 35488.5 & 15704.5 & 20452.8 & 18259.6 & 0.08 \\
\hline 500 & 124.7 & 0.028505 & 212670 & 39019.9 & 24000 & 21649.8 & 17013.6 & 0.08 \\
\hline 500 & 100.4 & 0.063803 & 140072 & 43729.8 & 24000 & 21736.5 & 11205.7 & 0.08 \\
\hline 500 & 73.1 & 0.184406 & 118914 & 66530.5 & 27000 & 50452.9 & 9513.1 & 0.08 \\
\hline 499 & 56.9 & 0.380303 & 95826 & 56621.5 & 28000 & 40467.4 & 6945.9 & 0.08 \\
\hline 500 & 40.4 & 0.872337 & 118387 & 66191.6 & 26843.5 & 64105.2 & 9471 & 0.072485 \\
\hline 499 & 20.6 & 2.70896 & 112982 & 59354.6 & 11000 & 86969.2 & 9038.6 & 0.08 \\
\hline
\end{tabular}


Table S1: Experimental conditions (mTorr, Temp, RH) of the analyzed data with the Integrated Areas $(\mathrm{Ox}, \mathrm{OH}, \mathrm{CO} 3, \mathrm{H} 2 \mathrm{O})$ of the fits. O 1s core level spectra were analyzed using XPST (free downloaded package) in Igor Pro. A Shirley background was subtracted before fitting with $\mathrm{L} / \mathrm{G}$ (a linear combination of Lorentzian and Gaussian) peaks. The fitting parameters (BE and FWHM of the peaks) are described in Table 1 of the main text. The average values and their variance were derived from unconstrained fits, and then constrained to be within the variance reported. The defect peak is kept at a constant area with respect to the bulk $\mathrm{O}_{\mathrm{x}}$ peak for the full $\mathrm{RH}$ range (rightmost column).

\section{Atomistic model of water adsorbed $\mathrm{TiO}_{2}$-terminayed STO}

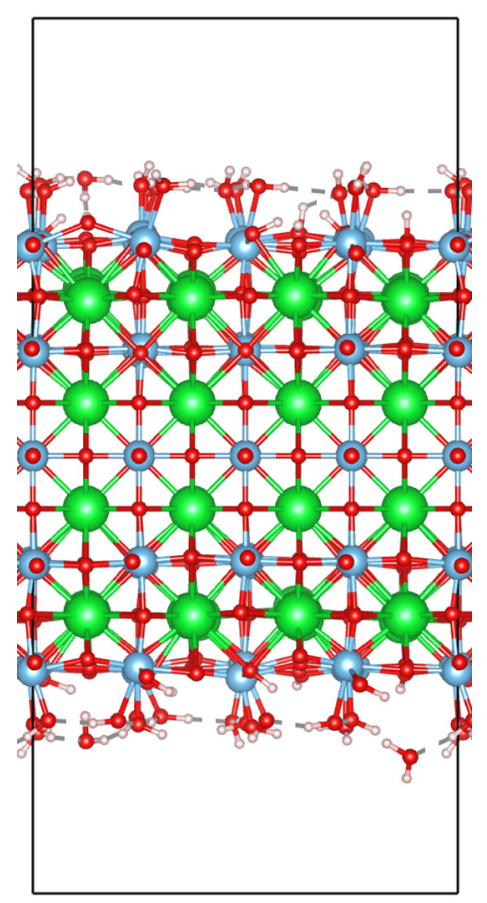

Figure S5 A 9-layer slab model of $\mathrm{TiO}_{2}$-terminayed STO employed for ab initio molecular dynamics simulations in this study is shown for the case of 1 monolayer water coverage. The lateral $4 \mathrm{x} 4$ supercell of STO features a total of $32 \mathrm{Ti}$ surface sites each of which can nominally adsorb a $\mathrm{H}_{2} \mathrm{O}$ molecule. The models considered in the study consist of up to a total of 464 atoms. $\mathrm{Sr}, \mathrm{Ti}, \mathrm{O}$ and $\mathrm{H}$ atoms are colored green, blue, red and white respectively. Structural visualizations were rendered using VESTA-3 (5).

\section{Choice of $\boldsymbol{U}_{\mathrm{o}-2 p}$ parameter on $\mathrm{O}$ sites within $\mathrm{PBE}+\boldsymbol{U}$}

$\mathrm{DFT}+U$ has been used to capture correlation effects in the valence band $\mathrm{O}-2 p$ states of oxide materials in numerous studies over the past decade and this practice is now well established (6-9). In particular, redox activity at metal oxide surfaces potentially entails hole-polaron formation involving $\mathrm{O}-2 p$ states $(10,11)$ and studies aimed at modeling such phenomena should employ either Hubbard- $U$ like on-site corrections(12) or accurate hybrid exchange-correlation functionals such HSE06(13). Here we employ a $\mathrm{PBE}+U$ scheme where the $U_{O-2 p}$ parameter on $\mathrm{O}$ sites is tuned to reproduce HSE06 adsorption and dissociation energies of water molecules on $\mathrm{TiO}_{2}$-terminated STO. Accordingly, we considered the non-dissociate adsorption energy of a single $\mathrm{H}_{2} \mathrm{O}$ molecule on a laterally $1 \times 1,9$-layer slab of $\mathrm{TiO}_{2}$-terminated $\mathrm{STO}$ by calculating the energy lowering as a water molecule moves towards the STO surface as shown in Figure S6. The total energy of the 
STO slab and incoming water molecule is calculated using HSE06 augmented by D3 dispersion corrections (HSE06-D3). The distance of the $\mathrm{H}_{2} \mathrm{O}$ molecule from the surface is constrained at different values by fixing the position of the $\mathrm{O}$ atom of the molecule while the rest of the atom positions are optimized at the HSE06-D3 level. At such constrained geometries, PBE $+U-\mathrm{D} 3$ energies are also calculated for different values of the $U_{O-2 p}$ parameter. Furthermore, at $1 \mathrm{ML}$ coverage the energy difference between molecular and dissociative adsorption modes of $\mathrm{H}_{2} \mathrm{O}$ on $\mathrm{TiO}_{2}$-terminated STO is also calculated with HSE06-D3 and PBE $+U$-D3 while varying $U_{o-2 p}$. We find that a value of $U_{O-2 p}=4 \mathrm{eV}$ represents an overall optimal choice reproducing both the adsorption and dissociation energies within $\sim 2 \%$ of HSE06-D3. Moreover, since $U_{O-2 p}=4 \mathrm{eV}$ is also sufficiently large to describe $\mathrm{O}-2 p$ hole polarons in oxides, we expect our parameterization to be transferrable across simulations of both ground state structural properties presented here as well as redox processes and associated energetics.
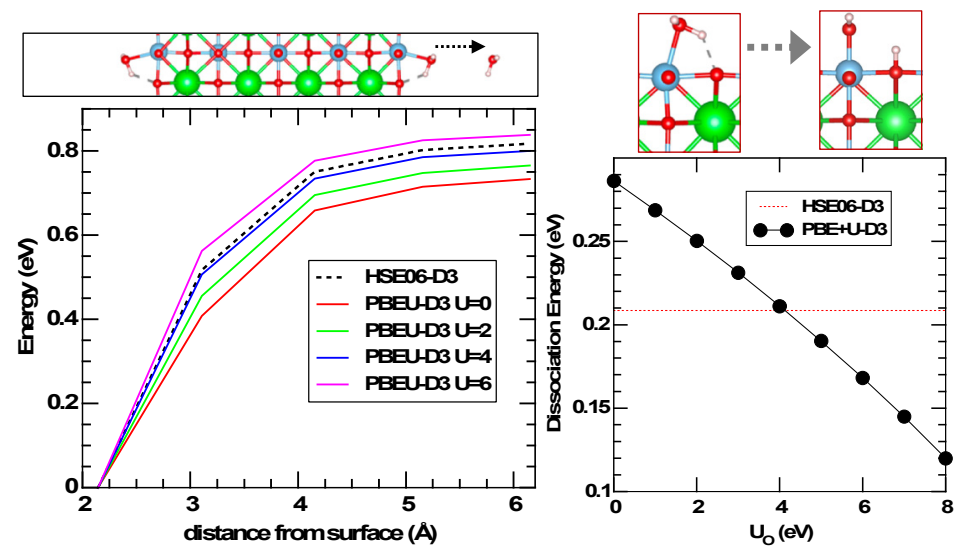

Figure S6 (left) Energy of adsorption of a water molecule at a TiO2-terminated STO surface plotted as a function of the distance of the molecule from the surface. HSE06-D3 energies (black dashed line) are compared against $\mathrm{PBE}+U$-D3 energies for different values of $U$ on $O-2 p$ orbitals. The energy at the HSE06-D3 equilibrium adsorption geometry is reference to zero in each case. (right) The $\mathrm{PBE}+U$-D3 energy difference between molecular and dissociative modes of adsorption of water at the STO surface is plotted for different choices of $U$ on $O-2 p$ orbitals. The corresponding HSE06-D3 result is indicated as a red horizontal line. It is seen that a choice of $U_{O}$ ${ }_{2 p}=4 \mathrm{eV}$ yields good agreement with HSE06-D3 for both adsorption and dissociation energies. 


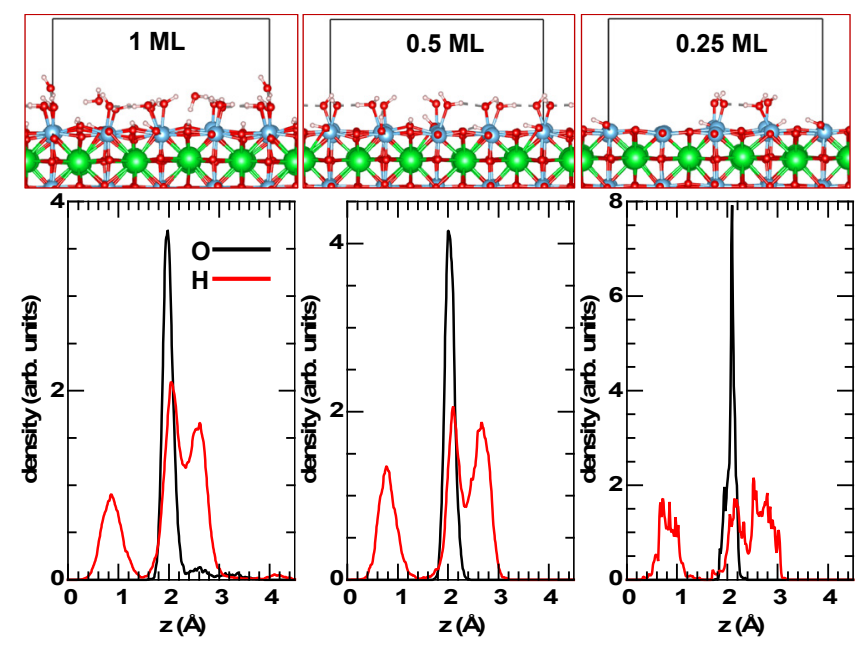

Figure S7 (Bottom) Calculated density profile of $\mathrm{O}$ (black line) and $\mathrm{H}$ (red line) atoms from adsorbed $\mathrm{H}_{2} \mathrm{O}$ and $\mathrm{OH}$ groups at the STO surface is shown as a function of distance $(\mathbf{z})$ along the normal from the terminating $\mathrm{TiO}_{2}$ layer. Results shown are obtained from 5 ps trajectories of 300 K AIMD runs for nominal $1 \mathrm{ML}, 0.5 \mathrm{ML}$ and $0.25 \mathrm{ML}$ coverages of $\mathrm{H}_{2} \mathrm{O}$. Representative snapshots at each coverage are shown at the top of the figure. It is apparent that at lower coverage a higher fraction of water molecules dissociate leading to a higher relative intensity of the $0.9 \AA \mathrm{H}$ peak. The $\mathrm{H}$ peak at $\sim 2 \AA$ is obtained primarily from intact $\mathrm{H}_{2} \mathrm{O}$ molecules including their H-bonding network, while the $\mathrm{H}$ peak at $\sim 2.6 \AA$ does not contribute to H-bonding. The relative weight of the $\sim 2.6 \AA \mathrm{H}$ peak is also higher at lower coverage again signifying a higher dissociated water fraction. Even though both Ti-adsorbed $\mathrm{H}_{2} \mathrm{O}$ and $\mathrm{OH}$ groups are present, the $\mathrm{O}$ density profile exhibits a relatively narrow distribution at $\sim 2 \AA$ from the surface owing to HO---OHO H-bonding. At low coverages the average $\mathrm{O}$ distance from the surface increases slightly towards $\sim 2.1 \AA$ as a higher fraction of dissociated waters leads to lower overall lower H-bonding.

\section{Decrease in FWHM of Ox Peak}

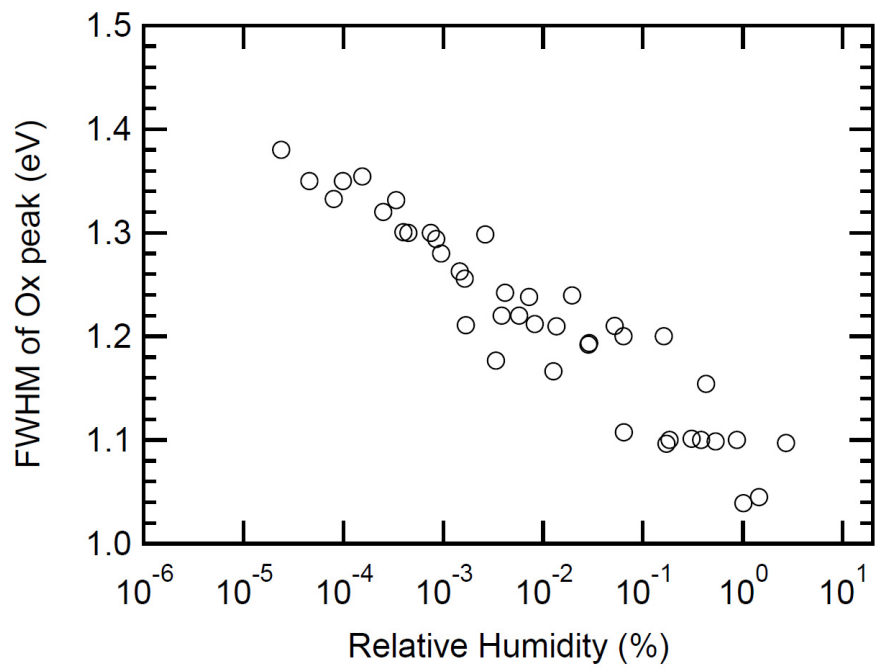

Figure S8. Plot of Ox FWHM (eV) from 500, 100, and 30 mTorr isobar data sets as a function of $\%$ RH. 


\section{Carbonate Determination/Sensitive Factor}

Figure S9 plots the $\mathrm{C}$ 1s spectra taken alongside the $\mathrm{O} 1 \mathrm{~s}$ spectra for the data presented in the main manuscript. The areas of the peaks within $286-290 \mathrm{eV}$ and assigned to $\mathrm{CO}_{3}$ were integrated. Figure S10 then plots the ratio of the integrated area of the orange peak within the $\mathrm{O}$ 1s spectra to this $\mathrm{C} 1 \mathrm{~s}$ peak area as a function of $\mathrm{RH}$. The ratio itself is due to the sensitivity factor for oxygen containing carbon species and is held fixed across RH. It was first determined by measuring the $\mathrm{O} 1 \mathrm{~s}: \mathrm{C}$ 1s XPS spectra of $\mathrm{CO}(\mathrm{g})$ at an incident energy of $735 \mathrm{eV}$ (same as these experimental conditions). For a $\mathrm{O}: \mathrm{C}$ ratio of $1: 1$, it was found to be $1.75: 1$. Therefore, for $\mathrm{CO}_{3}$, the ratio would be $3 \mathrm{x}, \sim 5.25: 1$.
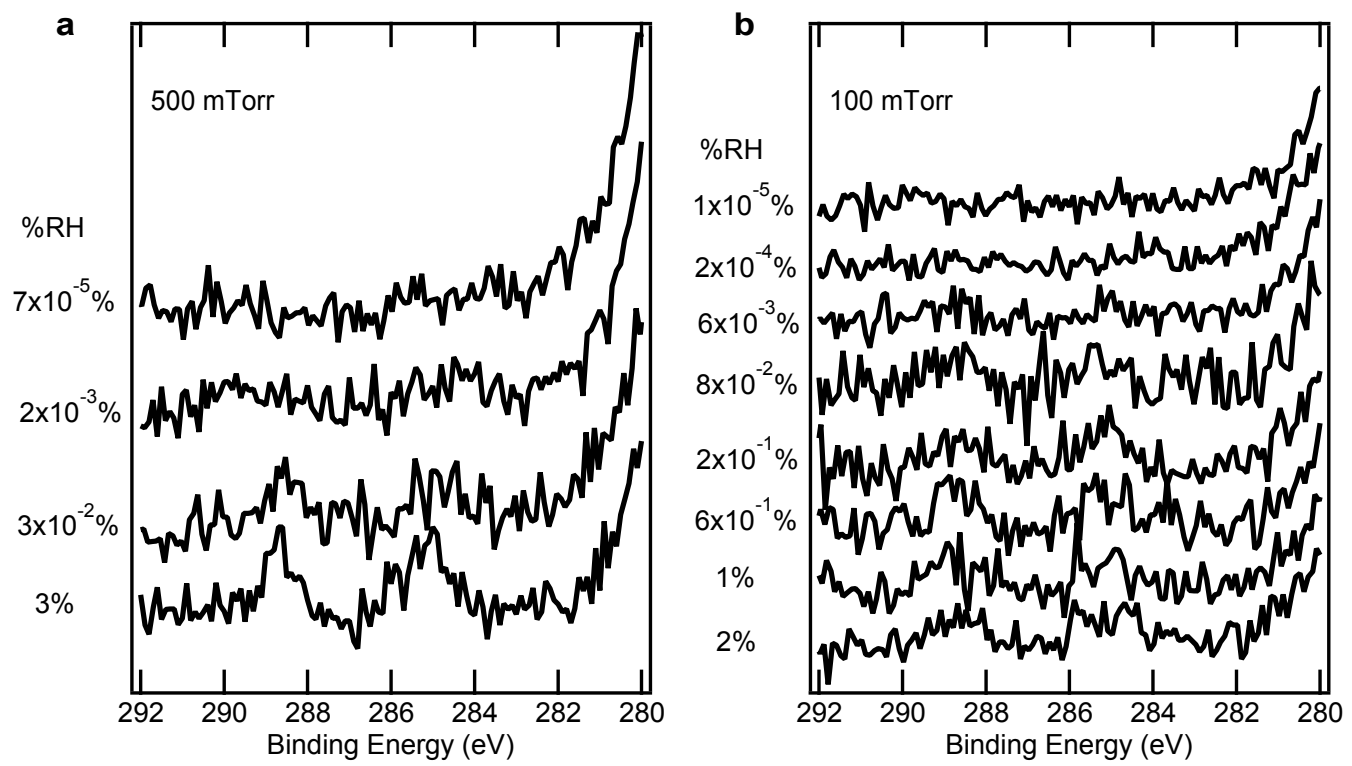

Figure S9. AP-C 1s spectra for a) $500 \mathrm{mTorr}$ isobar and b) $100 \mathrm{mTorr}$ isobar highlighting aliphatic carbon $(\sim 285 \mathrm{eV})$ and oxygen-containing carbon species $(\sim 289 \mathrm{eV})$ as a function of $\% \mathrm{RH}$.

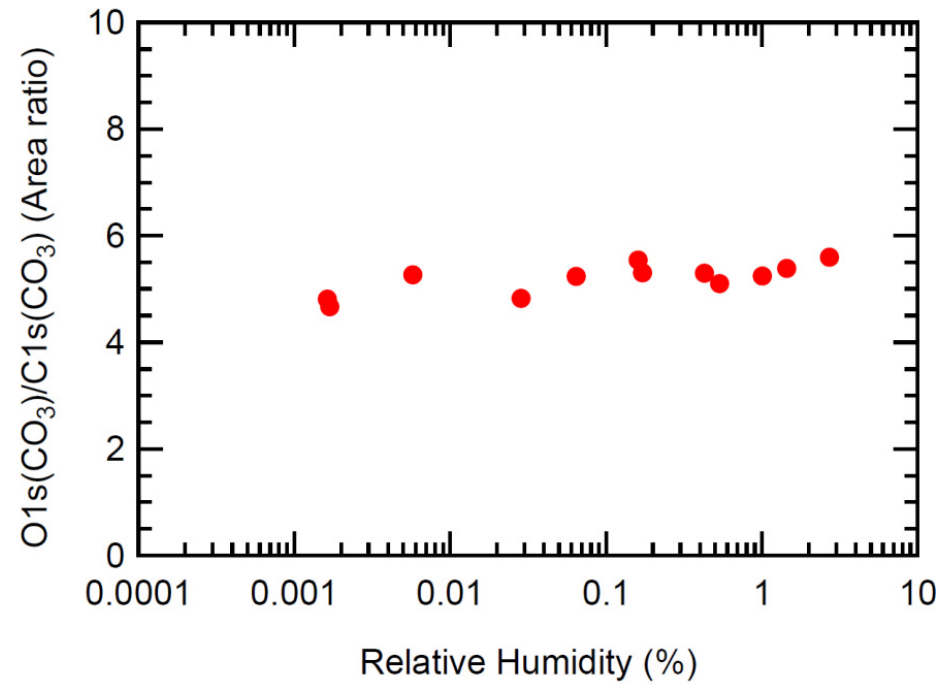

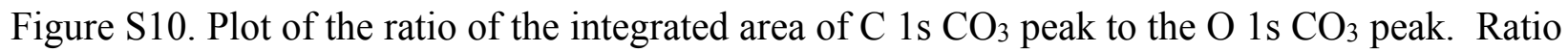
of $\mathrm{O} 1 \mathrm{~s} / \mathrm{C} 1 \mathrm{~s}$ integrated XPS peaks for carbonate species is $\sim 5.25$. 


\section{Results from fits without intrinsic defect: "hydroxylation" fitting procedure}

The following shows the results - each main manuscript figure and table that analyzes the raw data - using an alternative fitting model, without the intrinsic defect at UHV/high temperature conditions. Instead, the initial asymmetry of the O1s Ox peak is fit with the hydroxyl species, whose cross-section is increased with \%RH. These fits, for simplicity, are referred to as "hydroxylation" fits below.

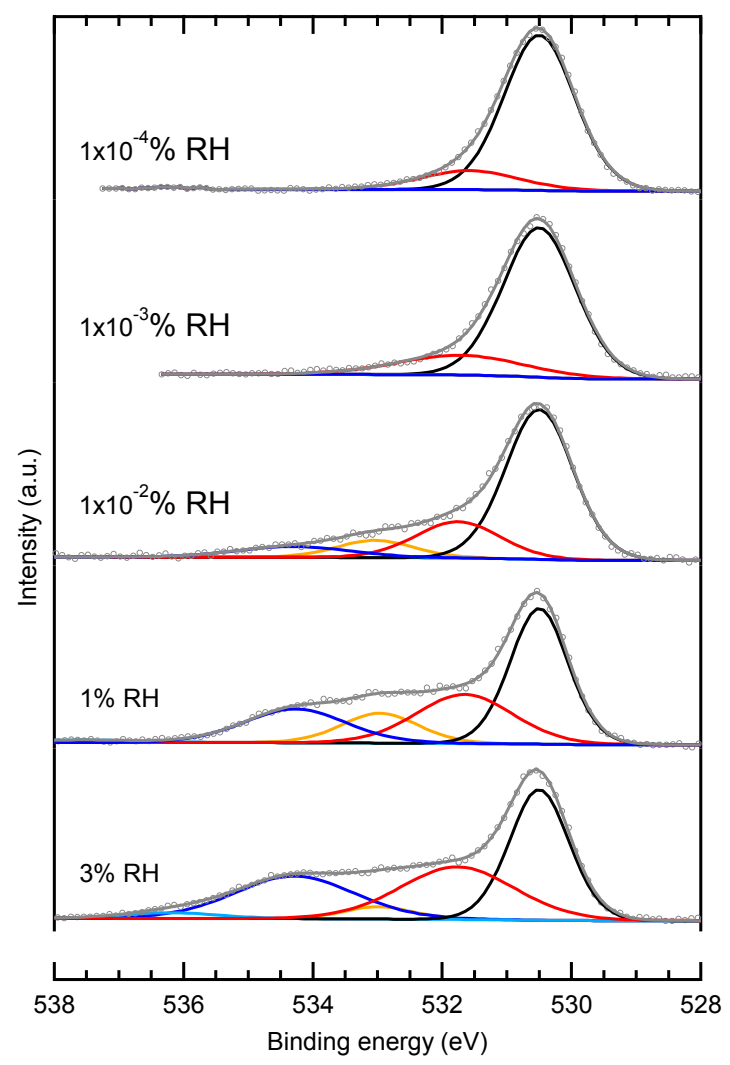

Figure S11 Figure 2 from the main manuscript for the "hydroxylation" fits.

\begin{tabular}{llll}
\hline & BE from Ox $(\mathrm{eV})$ & FWHM $(\mathrm{eV})$ & $\mathrm{L} / \mathrm{G}(\%)$ \\
\hline $\mathrm{Ox}$ & 530.5 & $1.4-1.0$ & 0.3 \\
$\mathrm{OH}$ & $532.1 \pm 0.2(1.06 \pm 0.2)$ & $1.9 \pm 0.4$ & 0.3 \\
$\mathrm{H}_{2} \mathrm{O}$ & $533.95 \pm 0.35(3.45 \pm 0.35)$ & $2.1 \pm 0.4$ & 0.3 \\
$\mathrm{CO}_{3}$ & $532.9 \pm 0.15(2.4 \pm 0.15)$ & $1.0 \pm 0.4$ & 0.3 \\
\hline
\end{tabular}

Table S2 Table 1 from the main manuscript for the hydroxylation fits. 

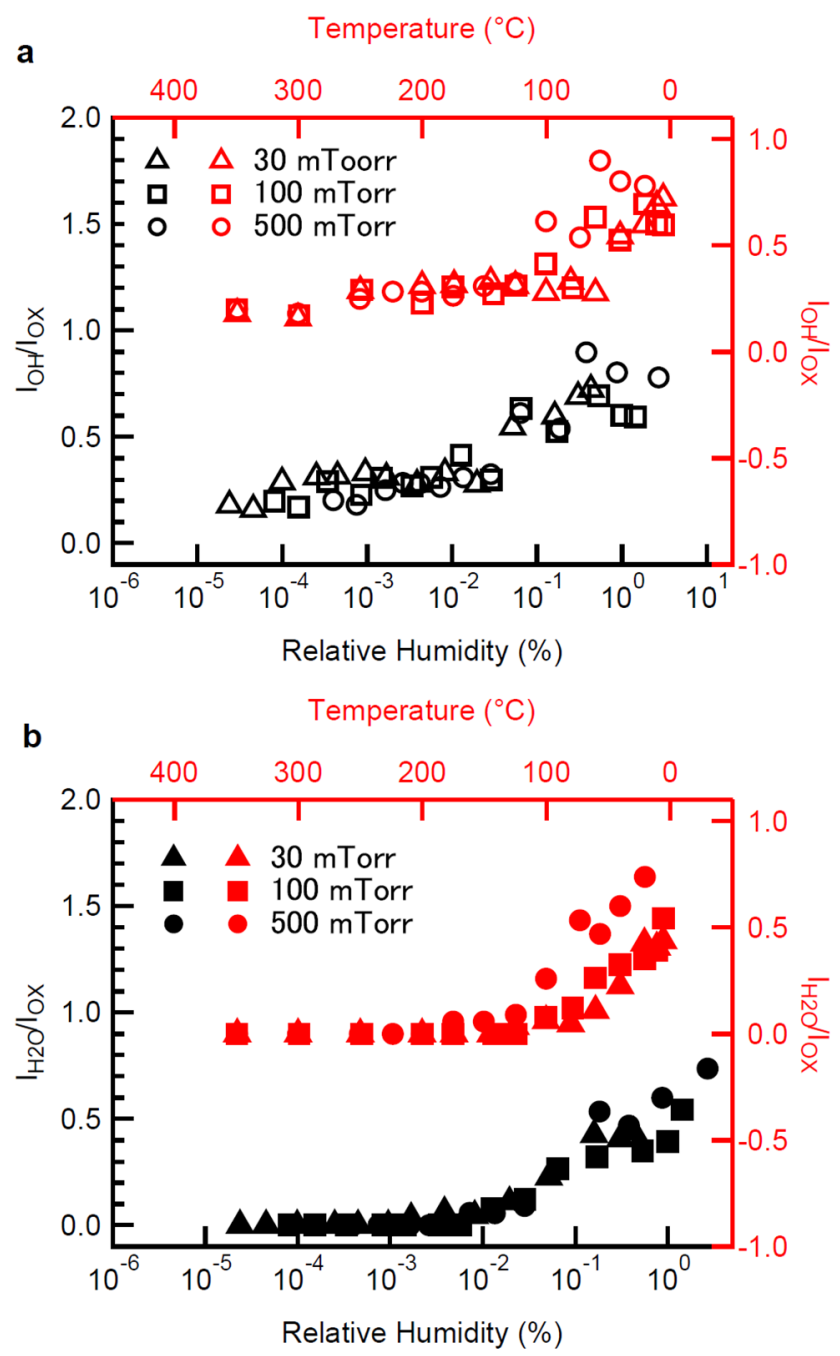

Figure S12 Figure 3 from the main manuscript for the "hydroxylation" fits: (a) IoH/Iox coverage and (b) IH2O/Iox coverage.

\section{Multilayer model details \& Comparison between two fittings}

The fitted data was further analyzed using a multilayer attenuation model. This model has been developed and used previous for multiple systems (14-16). The XPS intensity of component $i$ with a thickness $t_{i}$ can be calculated as

$$
I_{i}=D_{i} \beta_{i} \Phi_{i} N_{i} \sigma_{i} \int_{0}^{i} \exp \left(-\frac{z}{\lambda_{i}^{\prime}}\right) d z=D_{i} \beta_{i} \Phi_{i} N_{i} \sigma_{i} \lambda_{i}^{\prime}\left(1-\exp \left(-\frac{t_{i}}{\lambda_{i}^{\prime}}\right)\right)
$$

where $D$ is the spectrometer efficiency for a given KE, $\beta$ is the asymmetry parameter, $\Phi$ is the $\mathrm{X}$ ray photon flux, $N$ is the atomic density, $\sigma$ is the atomic photoionization cross section, and $\lambda^{\prime}=$ $\lambda^{*} \cos (\theta)$, where $\lambda$ is the inelastic mean free path (IMFP) of photoelectrons and $\theta$ is the emission angle of the photoelectrons from the surface normal ( $42^{\circ}$ in this work). Considering that we are only looking at $\mathrm{O} 1 \mathrm{~s}$ core spectra, the parameters $D, \beta, \Phi$, and $\sigma$ will be constant or cancel out when taking the ratio of different components, i.e. $\mathrm{Ox}, \mathrm{OH}$, and $\mathrm{H}_{2} \mathrm{O}$. The XPS intensity from adsorbed water, $\mathrm{IH}_{2} \mathrm{O}$, is therefore 


$$
I_{\mathrm{H}_{2} \mathrm{O}}=N_{\mathrm{H}_{2} \mathrm{O}} \lambda_{\mathrm{H}_{2} \mathrm{O}}^{\prime}\left(1-\exp \left(-\frac{t_{\mathrm{H}_{2} \mathrm{O}}}{\lambda_{\mathrm{H}_{2} \mathrm{O}} \mathrm{O}}\right)\right)
$$

The intensity from hydroxyl species, $I_{O H}$, has an additional term to account for the attenuation of the water over-layer

$$
I_{\mathrm{OH}}=N_{\mathrm{OH}} \lambda_{\mathrm{OH}}^{\prime}\left(1-\exp \left(-\frac{t_{\mathrm{OH}}}{\lambda_{\mathrm{OH}}^{\prime}}\right)\right) \exp \left(-\frac{t_{\mathrm{H}_{2} \mathrm{O}}}{\lambda_{\mathrm{H}_{2} \mathrm{O}}}\right)
$$

Similarly, the XPS signal from STO, IOx, will be attenuated by both $\mathrm{OH}$ and $\mathrm{H}_{2} \mathrm{O}$ species

$$
I_{O x}=N_{O x} \lambda_{O x}^{\prime} \exp \left(-\frac{t_{O H}}{\lambda_{O H}^{\prime}}\right) \exp \left(-\frac{t_{H_{2} O}}{\lambda_{H_{2} \mathrm{O}}^{\prime}}\right)
$$

The thickness of the $\mathrm{OH}$ layer, $t_{\mathrm{OH}}$, can be calculated from the intensity ratio of $\mathrm{OH}$ to $\mathrm{Ox}$,

$$
t_{O H}=\lambda_{O H}^{\prime} \ln \left[1+\frac{I_{O H}}{I_{O x}}\left(\frac{\lambda_{O x}^{\prime} N_{O x}}{\lambda_{O H}^{\prime} N_{O H}}\right)\right]
$$

Given that we are using a 3-site model (see main text), $N_{O H}=N_{O x}$. We also assume that $\lambda^{\prime} O H=$ $\lambda^{\prime} O x$, therefore Eq. 5 reduces to

$$
t_{O H}=\lambda_{O H}^{\prime} \ln \left[1+\frac{I_{O H}}{I_{O x}}\right]
$$

The thickness of the $\mathrm{H}_{2} \mathrm{O}$ layer, $t_{H_{2}} \mathrm{O}$, is similarly determined by taking the intensity ratio of $\mathrm{H}_{2} \mathrm{O}$ to $\mathrm{Ox}$

$$
t_{\mathrm{H}_{2} \mathrm{O}}=\lambda_{\mathrm{H}_{2} \mathrm{O}}^{\prime} \ln \left[1+\frac{I_{\mathrm{H}_{2} \mathrm{O}}}{I_{\mathrm{OH}}}\left(\frac{\lambda_{\mathrm{OH}}^{\prime} N_{\mathrm{OH}}}{\lambda_{H_{2} \mathrm{O}}^{\prime} N_{H_{2} \mathrm{O}}}\right)\left(1-e^{-t_{\mathrm{OH}} / \lambda_{\mathrm{OH}}^{\prime}}\right)\right]
$$

The atomic density for the oxide layer and hydroxyl layer are obtained from the bulk density of STO $\left(5.1 \mathrm{~g} \mathrm{~cm}^{-3}\right), N_{O x}=N_{O H}=50.2$ oxygen $\mathrm{nm}^{-3}$, and $N_{H 2 O}=33.4$ oxygen $\mathrm{nm}^{-3}$. The IMFP for STO $\left(\lambda^{\prime} O x\right)$ was determined from the NIST Standard Reference Database 71 software v1.1(17) using the TPP-2M predictive formula equation and a bandgap of $3.25 \mathrm{eV}$ and valence electron count of 24. The IMFP for water was taken from (15). 

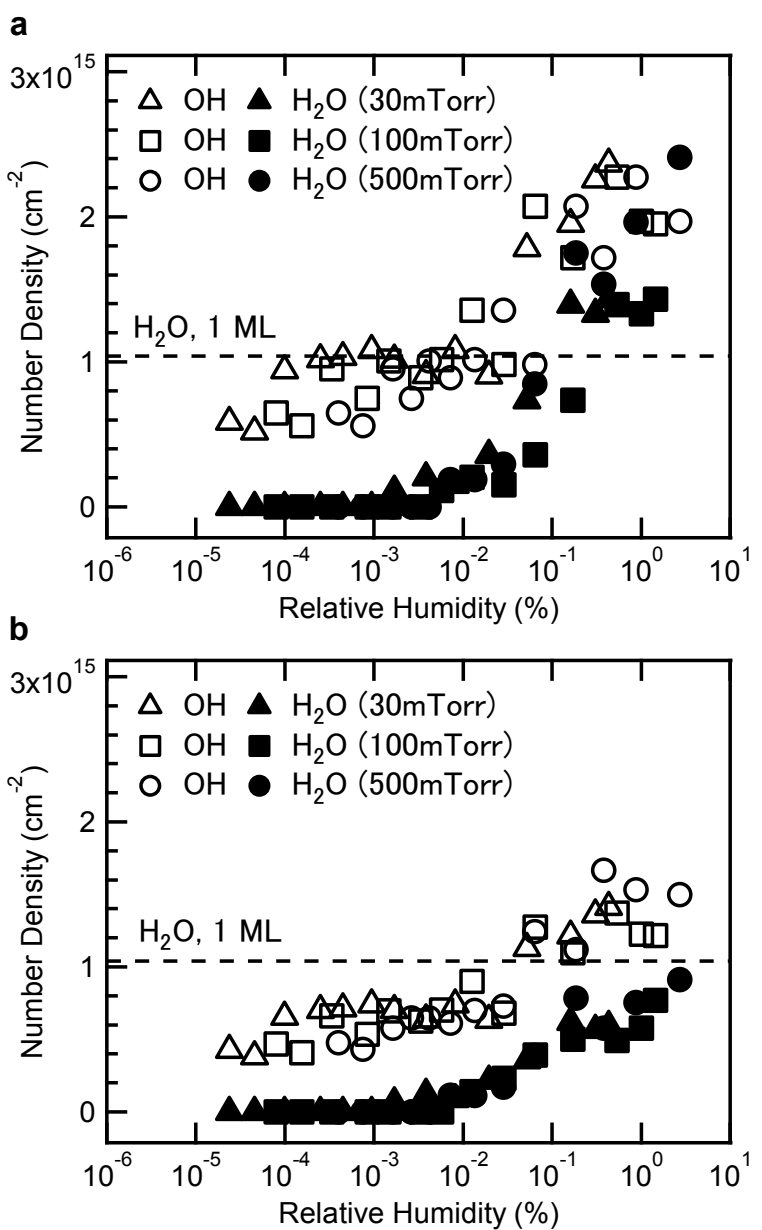

Figure S13 Figure 4 from the main manuscript for the "hydroxylation" fits: (a) sub-monolayer model and (b) multi-layer model

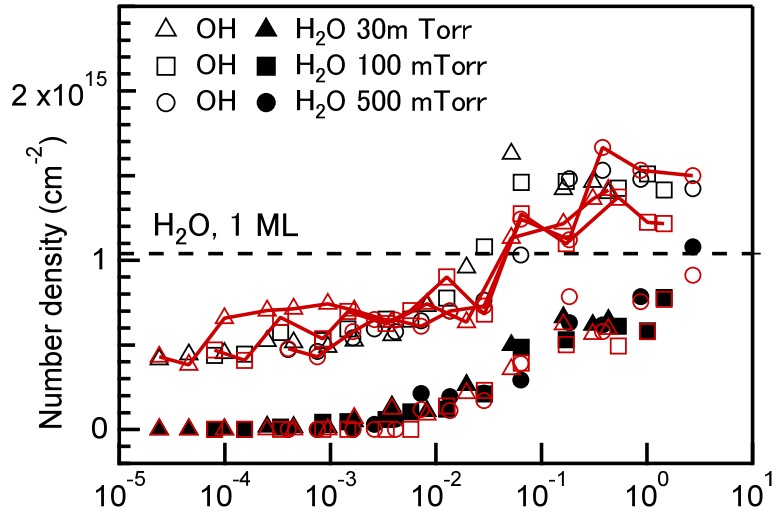

Relative Humidity (\%)

Figure S14 A comparison of the "hydroxylation" fits (red symbols) to the fits in the main text (black symbols) for the final $\mathrm{OH}, \mathrm{H}_{2} \mathrm{O}$ coverages determined by the multi-layer model. In order to make the agreement shown, the $\mathrm{OH}$ coverage determined from the intrinsic defect fits was shifted up by a constant $3.25 \times 10^{14} \mathrm{~cm}^{-2}$ across $\mathrm{RH}$. The $\mathrm{H}_{2} \mathrm{O}$ coverage derived for both fits is as shown. 


\section{Above surface density $p$}

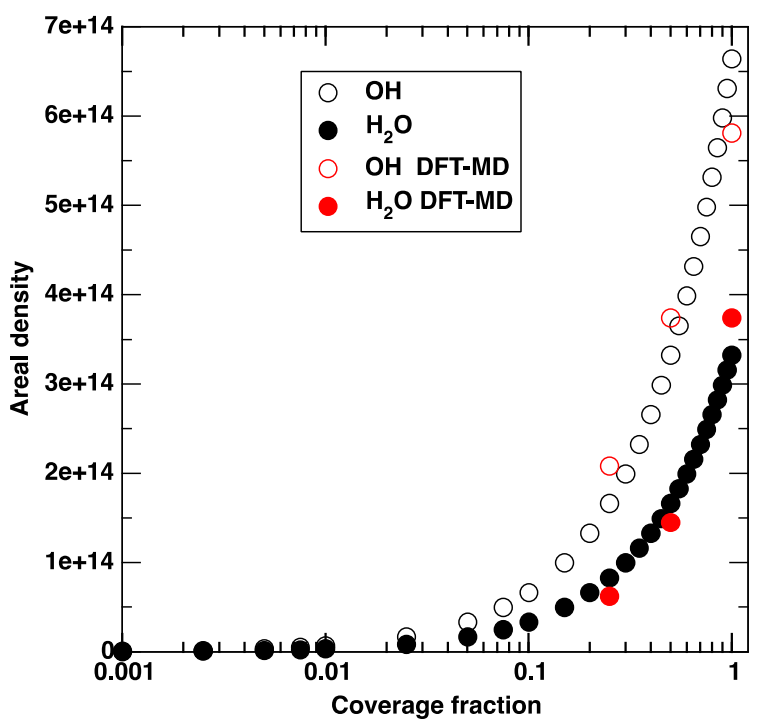

Figure S15 Schematic illı

of coverage. Black circles correspond to the case where one assumes only the partially dissociative C-III configuration to be present at all coverages. Red circles indicate DFT AIMD results. At $1 / 4$ and $1 / 2$ ML coverages, the dissociative $\mathrm{C}$-II configuration is accessible via entropic effects which leads to a higher (lower) fraction of $\mathrm{OH}\left(\mathrm{H}_{2} \mathrm{O}\right)$ relative to the $\mathrm{C}$-III configuration. At higher $1 \mathrm{ML}$ coverage the non-dissociative $\mathrm{C}$-I configuration is stable while the $\mathrm{C}$-III configuration is accessed through thermal fluctuations. Thus a higher fraction of intact $\mathrm{H}_{2} \mathrm{O}$ results.

\section{Additional comparisons between theoretical calculations and experimental results}

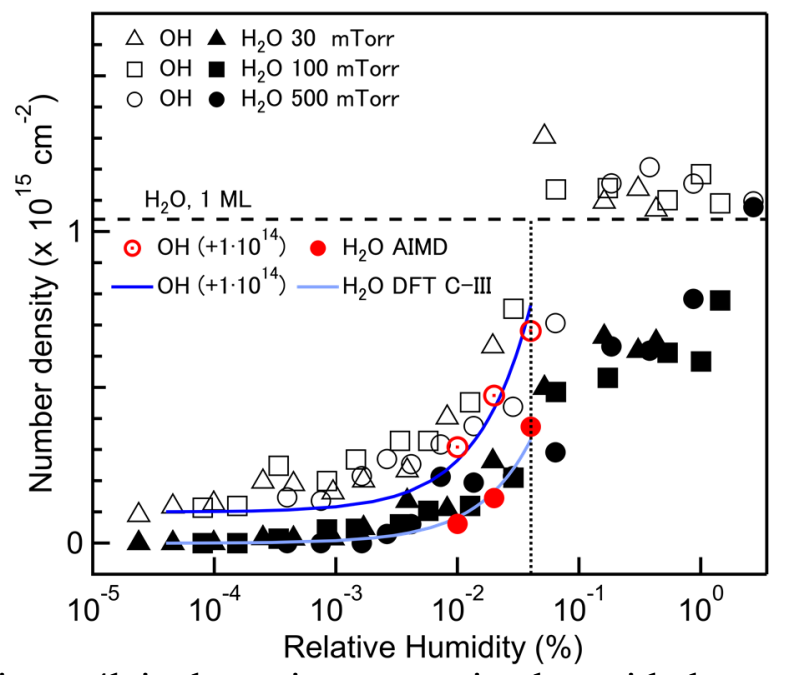

Figure S16 Replica of Figure 4b in the main manuscript, but with the scaling between ML coverage in theory and $\mathrm{RH}$ in experiment, such that $1 \mathrm{ML}$ in theory (equivalent $\mathrm{H}_{2} \mathrm{O}$ to $\mathrm{Ti}$ ) sites occurs at $4 \times 10^{-2} \mathrm{RH}$ as opposed to $2 \times 10^{-2} \mathrm{RH}$. Both the AIMD and the C-III configuration are scaled the same way. The $\mathrm{OH}$ coverage for both is offset by $1 \times 10^{14} \mathrm{~cm}^{-2}$, as in the main manuscript. 


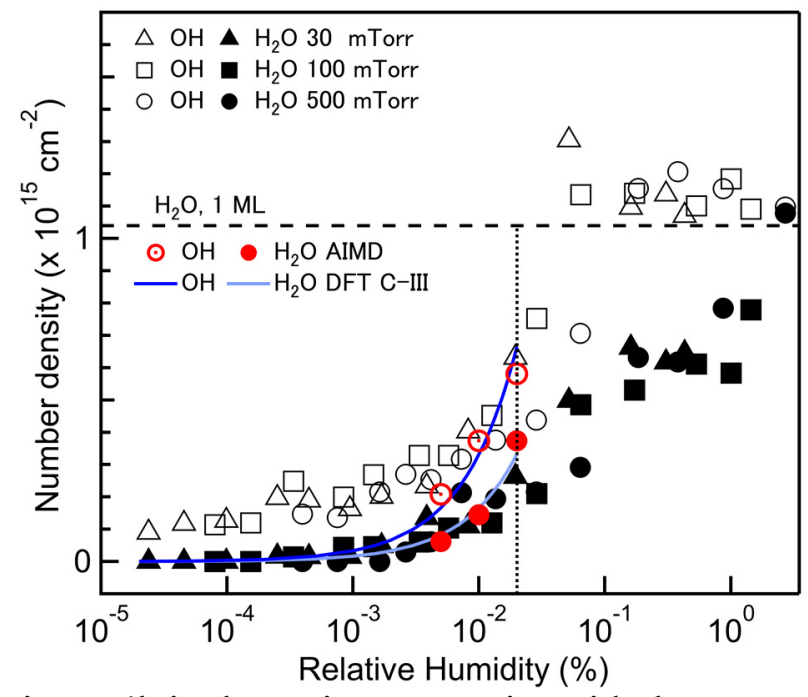

Figure S17 Replica of Figure 4b in the main manuscript with the same scaling between theory and experiment, but without any offset to the $\mathrm{OH}$ coverage derived from the theory.

\section{References}

1. E. Heifets, S. Dorfman, D. Fuks, E. Kotomin, A. Gordon, Atomistic simulation of SrTiO3(001) surface relaxation. J. Phys.-Condes. Matter 10, L347-L353 (1998).

2. M. S. Martin-Gonzalez et al., In situ reduction of (100) SrTiO3. Solid State Sci. 2, 519524 (2000).

3. S. A. Chambers, T. C. Droubay, C. Capan, G. Y. Sun, Unintentional F doping of SrTiO3(001) etched in HF acid-structure and electronic properties. Surf. Sci. 606, 554558 (2012).

4. T. Ohsawa, R. Shimizu, K. Iwaya, S. Shiraki, T. Hitosugi, Negligible Sr segregation on SrTiO3(001)-( 13×13)-R33. $7^{\circ}$ reconstructed surfaces. Appl. Phys. Lett. 108, 161603 (2016).

5. Momma, K.; Izumi, F. VESTA 3 for Three-Dimensional Visualization of Crystal, Volumetric and Morphology Data. J. Appl. Crystallogr. 2011, 44 (6), 1272-1276.

6. Droghetti, A.; Pemmaraju, C. D.; Sanvito, S. Polaronic Distortion and Vacancy-Induced Magnetism in MgO. Phys. Rev. B 2010, 81 (9), 092403.

7. Garcia-Lastra, J. M.; Myrdal, J. S. G.; Christensen, R.; Thygesen, K. S.; Vegge, T. DFT+U Study of Polaronic Conduction in Li2O2 and Li2CO3: Implications for Li-Air Batteries. J. Phys. Chem. C 2013, 117 (11), 5568-5577.

8. Ji, Y.; Wang, B.; Luo, Y. Location of Trapped Hole on Rutile-TiO 2 (110) Surface and Its Role in Water Oxidation. J. Phys. Chem. C 2012, 116 (14), 7863-7866.

9. Geneste, G.; Amadon, B.; Torrent, M.; Dezanneau, G. DFT+ U Study of Self-Trapping, Trapping, and Mobility of Oxygen-Type Hole Polarons in Barium Stannate. Phys. Rev. B 2017, 96 (13), 134123.

10. Chen, X.; Choing, S. N.; Aschaffenburg, D. J.; Pemmaraju, C. D.; Prendergast, D.; Cuk, $\mathrm{T}$. The Formation Time of $\mathrm{Ti}-\mathrm{O}^{*}$ and $\mathrm{Ti}-\mathrm{O}^{*}-\mathrm{Ti}_{\text {Radicals at }}$ the $\mathrm{n}-\mathrm{SrTiO}_{3} /$ Aqueous Interface during Photocatalytic Water Oxidation. J. Am. Chem. Soc. 2017, 139 (5), 18301841 . 
11. Herlihy, D. M.; Waegele, M. M.; Chen, X.; Pemmaraju, C. D.; Prendergast, D.; Cuk, T. Detecting the Oxyl Radical of Photocatalytic Water Oxidation at an N-SrTiO3/Aqueous Interface through Its Subsurface Vibration. Nat. Chem. 2016, 8 (6), 549-555.

12. Erhart, P.; Klein, A.; Åberg, D.; Sadigh, B. Efficacy of the DFT + U Formalism for Modeling Hole Polarons in Perovskite Oxides. Phys. Rev. B 2014, 90 (3), 035204.

13. Krukau, A. V.; Vydrov, O. A.; Izmaylov, A. F.; Scuseria, G. E. Influence of the Exchange Screening Parameter on the Performance of Screened Hybrid Functionals. J. Chem. Phys. 2006, 125 (22), 224106.

14. S. Yamamoto et al., Water Adsorption on alpha-Fe2O3(0001) at near Ambient Conditions. J. Phys. Chem. C 114, 2256-2266 (2010).

15. J. T. Newberg et al., Autocatalytic Surface Hydroxylation of $\mathrm{MgO}(100)$ Terrace Sites Observed under Ambient Conditions. J. Phys. Chem. C 115, 12864-12872 (2011).

16. J. T. Newberg et al., Formation of hydroxyl and water layers on $\mathrm{MgO}$ films studied with ambient pressure XPS. Surf. Sci. 605, 89-94 (2011).

17. C. J. Powell, A. Jablonski, F. Salvat, NIST databases with electron elastic-scattering cross sections, inelastic mean free paths, and effective attenuation lengths. Surface and Interface Analysis 37, 1068-1071 (2005). 